Article

\title{
Cross-Sectional Performance of Hollow Square Prisms with Rounded Edges
}

\author{
Hiroyuki Shima ${ }^{1, *} \mathbb{C}$, Nao Furukawa ${ }^{2}$, Yuhei Kameyama ${ }^{3}$, Akio Inoue ${ }^{4}$ and Motohiro Sato ${ }^{5, *}$ \\ 1 Department of Environmental Sciences, University of Yamanashi, Kofu, Yamanashi 400-8510, Japan \\ 2 Department of Socio-Environmental Engineering, School of Engineering, Hokkaido University, Sapporo, \\ Hokkaido 060-8628, Japan; ponkawa@eis.hokudai.ac.jp \\ 3 Division of Engineering and Policy for Sustainable Environment, Graduate School of Engineering, \\ Hokkaido University, Sapporo, Hokkaido 060-8628, Japan; ab884yuheidef10@eis.hokudai.ac.jp \\ 4 Faculty of Agriculture, Kindai University, Nara 631-8505, Japan; iakio@nara.kindai.ac.jp \\ 5 Division of Mechanical and Aerospace Engineering, Faculty of Engineering, Hokkaido University, \\ Sapporo 060-8628, Japan \\ * Correspondence: hshima@yamanashi.ac.jp (H.S.); tayu@eng.hokudai.ac.jp (M.S.)
}

Received: 21 May 2020; Accepted: 8 June 2020; Published: 11 June 2020

\begin{abstract}
Hollow-section columns are one of the mechanically superior structures with high buckling resistance and high bending stiffness. The mechanical properties of the column are strongly influenced by the cross-sectional shape. Therefore, when evaluating the stability of a column against external forces, it is necessary to reproduce the cross-sectional shape accurately. In this study, we propose a mathematical method to describe a polygonal section with rounded edges and vertices. This mathematical model would be quite useful for analyzing the mechanical properties of plants and designing plant-mimicking functional structures, since the cross-sections of the actual plant culms and stems often show rounded polygons.
\end{abstract}

Keywords: square bamboo; structural mechanics; buckling; bending; hollow columns; biomimetics

\section{Introduction}

Hollow columns are an excellent functional form as Galileo first reported in the 17th Century [1]. They exhibit superior mechanical stability and strength against axial compression, bending, and torsion compared to solid columns with the same cross-sectional area. This mechanical advantage is mainly due to the improved cross-sectional performance caused by the presence of the internal cavity. In the case of hollow columns, the constituent material is absent in the cavity; instead, it is distributed far from the columnar axis. This material distribution increases the buckling resistance and bending stiffness of the column and reduces the maximum bending stress at the outer edges. In addition to increased rigidity and strength, hollow columns are also superior to solid columns in that they are lightweight and material-saving. In this way, hollow cylinders and prisms have been used for a long time as building members and machine parts because they have excellent cross-sectional performance and are economical (require little material).

Besides artificial structures, the culms, stems, and branches of some wild plants have an excellent functional form with hollowness. Bamboo is a salient example of such plants [2,3], native to warm and moist tropical regions in the world [4,5]. From a structural mechanics perspective, bamboo culms are considered long hollow cylinders with a slightly thicker base and thinner tips [6]. This tapered configuration improves rigidity against bending forces caused by cross winds compared to hollow cylinders of uniform diameter. Furthermore, due to its hollow nature, bamboo culms are lighter than solid columns with the same culm diameter and therefore can grow faster with a small amount of 
photosynthates $[7,8]$. This fast-growing feature allows bamboo to compete favorably for survival with other plants. These facts prove that bamboo is a plant with an excellent functional structure that combines light weight, high stability, and material saving.

Let us remind that many species of bamboo are endowed with circular cross-sections. Nevertheless, certain species of bamboo, like Chimonobambusa quadrangularis (Franceschi) Makino, have a square-like cross-section along the whole length of the culms [9]. Figure 1a shows the cross-section of the square bamboo [10]. It is typically two or three centimeters in outer diameter [10], having a square cross-section with rounded sides and filleted corners, as schematically illustrated by Figure 1b. Besides the square bamboo, many plants are known to have polygon-shaped cross-sections in their stems or branches [11]. A mint (Lamiaceae) showing a square stem and a papyrus (Cyperus microiria) showing a triangular stem are cases in point; see Figure 1c,d. From the point of view of plant physiology, we speculate that the reason why these plants have a polygonal cross-section is that this may promote the formation of phyllotaxis and help identify the location of leaf formation. On the other hand, the choice of polygonal shapes instead of a simple circular annulus is expected to provide a feasible shift in their cross-sectional performance, though no quantitative examination on this issue has been found. Moreover, the polygonal cross-section of the plant has significantly rounded sides and vertices, unlike the exact regular polygon, which consists of straight line segments and sharp vertices. Therefore, the simple mathematical formulas established in the engineering field cannot be applied to the evaluation of sectional performance. To address this issue, it is essential to develop a theoretical model that describes plant-like polygons with rounded sides and filleted corners.

(a)

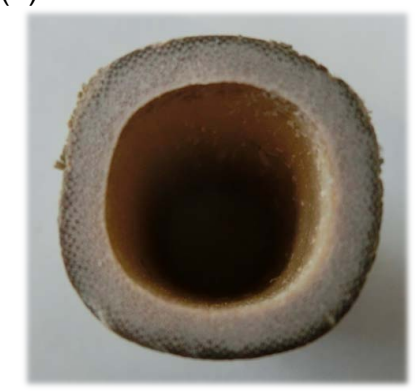

(c)

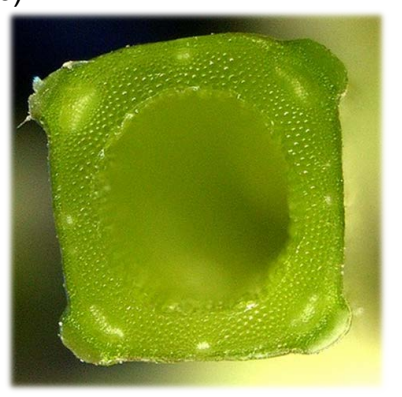

(b)

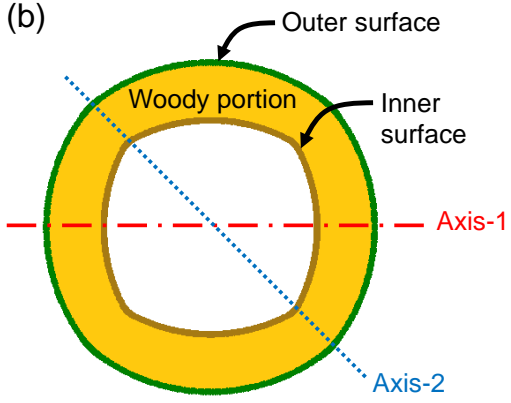

(d)

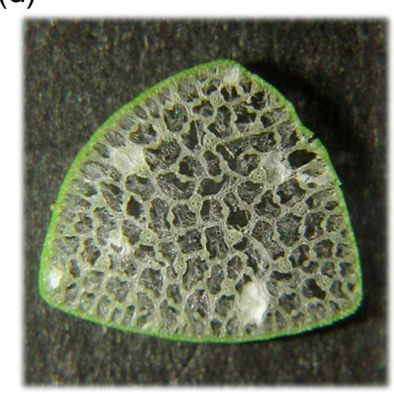

Figure 1. (a) Photo of the cross-section of a square bamboo: Chimonobambusa quadrangularis (Franceschi) Makino. (b) Approximate curve of the square bamboo's cross-section. (c) Square cross-section of the mint stem; Lamium album var. barbatum [12]. (d) Triangular cross-section of the papyrus stem; Cyperus microiria [13].

In the present work, we create a mathematical model that describes the rounded squares observed in the cross-section of a square bamboo. By varying the parameters, this model is broadly applicable to polygonal hollow (and solid) prism and can also include triangular, pentagonal, and hexagonal prisms. This model is used to analyze the cross-sectional performance of hollow square prisms. 
Particular emphasis is placed on the section modulus and radius of gyration of the area, which generally determine the mechanical stability and strength of the prism.

\section{Modeling a Rounded Square with Filleted Corners}

We propose a quadrangular prismatic model whose shape is similar to that of square bamboo. Figure 2 illustrates the drawing of the cross-section, which possesses the four-fold symmetry composed of four long circular arcs (colored in magenta) and four short circular arcs (blue). The cross-sectional shape is uniquely determined by the set of three geometric parameters: $\{\ell, \theta, h\}$, as explained below. By arranging two of the closed curves with in a different concentric manner, the hollow cross-section as depicted in Figure $1 \mathrm{~b}$ can be obtained.

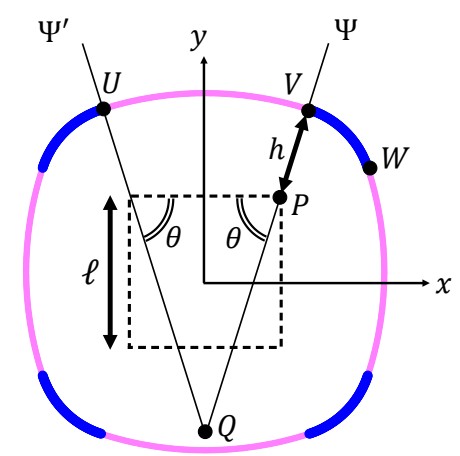

Figure 2. A rounded square model with a four-fold symmetry. Schematic definitions of the three parameters, $\ell, \theta$, and $h$ are shown.

To obtain the model, we first prepare a reference square with the side length $\ell$, which is depicted by a dotted square in Figure 2. Next, we draw a straight line $\Psi$ that passes through the upper-right vertex, $\mathrm{P}$, of the reference square such that it forms an angle $\theta$ with the horizontal side of the square. Another straight line $\Psi^{\prime}$ is also drawn at the symmetric position to the line $\Psi$ with respect to the $y$-axis. The intersection of the two lines is denoted by $Q$. Then, we draw a circular arc around $Q$, which is represented by UV in Figure 2.

The radius of the circular sector UVQ is defined by the sum of the line segment length PQ and $h$, as indicated in the figure. Another circular arc around $\mathrm{P}$ with radius $h$, represented by VW in Figure 2, is also drawn. Finally, we perform a similar drawing of circular arcs as above for the other three vertices of the reference square to create the rounded square cross-section with filleted corners.

The variable range of the angle $\theta$ is defined to be $\pi / 4 \leq \theta \leq \pi / 2$. In particular, when $\theta=\pi / 4$, the small arc VW converges to a point so that the rounded square becomes a true circle with a radius of $(\ell / \sqrt{2})+h$. On the other hand, when $\theta=\pi / 2$, the large arc $\mathrm{UV}$ is a horizontally straight line segment, and VW becomes a quadrant arch.

The total area enclosed by the rounded square, designated by $A$, is a function of the three parameters $\ell, \theta$, and $h$. Unless $\theta=\pi / 2$, it is explicitly written as:

$$
A(\ell, \theta, h)=(2 \pi-4 \theta)\left(\frac{\ell}{2 \cos \theta}+h\right)^{2}+(1-\tan \theta) \ell^{2}+(4 \theta-\pi) h^{2}
$$

Only when $\theta=\pi / 2$, Equation (1) does not work as $(\cos \theta)^{-1}$ and $\tan \theta$ diverge; instead, it is replaced by:

$$
A\left(\ell, \theta=\frac{\pi}{2}, h\right)=\pi h^{2}+4 \ell h+\ell^{2} .
$$

Note that the model possesses the tangent continuity at any point on the closed curve. In addition, the model is essentially different from super-ellipses $[14,15]$, being expressed in terms of the $p$-norm and the vector notation by $\left\|\boldsymbol{r}-\boldsymbol{r}_{0}\right\|_{p}=$ const, or its generalization called a Gielis curve [16-18], while both 
of them are known as powerful tools for describing the natural shape of plants. Furthermore, a method of drawing a rounded triangle by using a complex function has been proposed quite recently [19], but again, our method is essentially different from it.

A notable feature of the modeling we have presented is based on a patchwork-like method, where four pairs of long and short arcs are sequentially jointed to form a closed curve of a rounded square. Due to this feature, it is possible to introduce asymmetry into the closed curve, as will be discussed later. In other words, we can change the curvature of the sides of the polygon and the degree of rounding of the vertices at each location. In addition, the constituent arcs are represented by simple functions, making it easy to calculate exact solutions for cross-sectional performance. These features are expected to be quite useful when analyzing the mechanical properties of polygonal culms and stems that actually exist in nature.

It should be also emphasized that attempts to reproduce the complex morphology of plants mathematically have great academic significance [20-24] and high applied value from the perspective of biomimetic technology $[25,26]$. In this respect, the mathematical model proposed in this paper can be expected to contribute to obtain better understandings of the mechanical properties of plants with polygonal culms and stems and to develop the plant-mimetic optimal design of high-rise buildings and hollow pipe structures.

Figure 3a shows how the geometry varies with changing the values of $\ell$ and $\theta$. In the drawing, we fixed the area to be constant as $A=\pi a_{0}^{2}$, and the constant $a_{0}$ was used as the unit of length in the model. Under such a constant-area condition, $h$ is not an independent variable, but a function of $\ell$ and $\theta$, as follows from Equations (1) and (2). Figure $3 b$ shows the dependence of $h$ on $\theta$ for various values of $\ell$, indicating that $h$ increases monotonically with $\theta$ for every $\ell$. In particular, when $\ell=\sqrt{2} a_{0}$, the curve passes through the point $(h, \theta)=(0, \pi / 4)$. For larger $\ell$, the $h$-curve has an intersection with the $\theta$-axis at $\theta=\theta^{*}(>\pi / 4)$. As a result, the definition range of $\theta$ becomes limited to $\theta^{*} \leq \theta \leq \pi / 2$ with the lower limit $\theta^{*}$, which is an increasing function of $\ell$. Eventually, when $\ell=\sqrt{\pi} a_{0}, \theta^{*}$ reaches $\pi / 2$ so that the curve shrinks to a point located at $(h, \theta)=(0, \pi / 2)$, that represents a strict quadrangle with four right-angle corners.

(a)
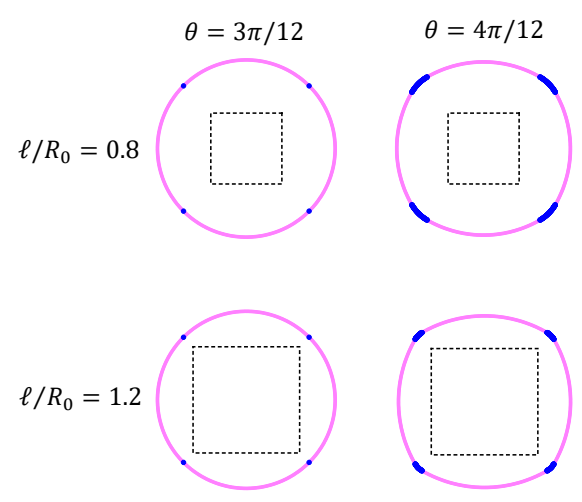
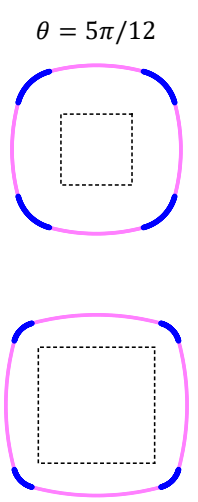

(b)

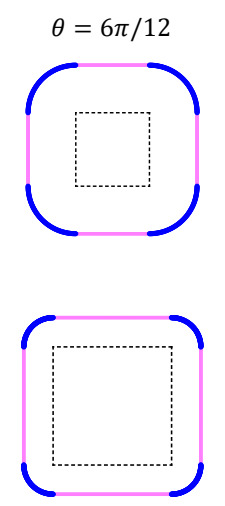

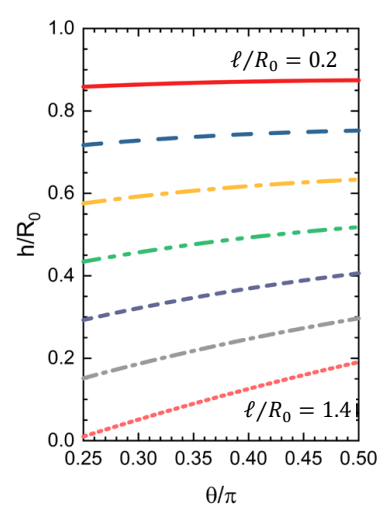

Figure 3. (a) Geometric variation of a rounded square due to changes in the variables $\theta$ and $\ell$ under the condition where the enclosed area $A=\pi a_{0}^{2}$ is fixed. The constant $a_{0}$ serves as the unit of length. Dotted lines show the reference squares with side length $\ell$. (b) Dependence of the small sector's radius $h$ on $\theta$. The value of $\ell / a_{0}$ is varied with an interval of 0.2 .

Two square closed curves with rounded sides and filleted corners, drawn by the above-mentioned method, are arranged in a double concentric manner to create an approximate curve that resembles the cross-section of hollow square prisms found in square bamboos and other plants. An example is shown in Figure $1 b$. The outer and inner closed curves that enclose the woody portion of the square bamboo are constructed by the parameter settings: $\ell=1.0, \theta=6 \pi / 20$, and $h=0.6$ for the outer curve 
and $\ell=1.0, \theta=7 \pi / 20$, and $h=0.2$ for the inner curve, respectively, with a common unit of length $a_{0}$. In the case of actual square bamboo, the shape of the cross-section changes depending on the height from the ground to the tip. We confirmed that many of them can be reproduced by setting appropriate parameter values $\{\ell, \theta, h\}$.

\section{Cross-Sectional Performance}

Our current aim is to evaluate the performance of rounded square cross-sections observed in square bamboo culm and other plant stems. Generally, for a given column, the cross-section performance measures the degree of contribution from the cross-sectional shape to the mechanical stability and strength of the column against external forces. It is characterized by four morphological quantities evaluated from the cross-sectional shape: the area $A$, the second moment of the area $I$, the gyration radius of the area $R_{g}=\sqrt{I / A}$, and the section modulus $Z=I / e$. Here, $e$ is the greatest distance from an axis assigned to the cross-section to the extreme edge of the outer enclosing curve.

The following discussion will focus on how the latter two quantities, $R_{g}$ and $Z$, depend on the geometry of the cross-section, because the two are directly relevant to the mechanical stability and strength of the column, respectively. In plain words, $R_{g}$ measures the buckling resistance of a column under axial compression, and $Z$ measures the yielding strength of it; these points will be revisited later. The actual calculation method of $R_{g}$ and $Z$ will be explained in Appendices A and B. It should be noted that, in general, the values of $R_{g}$ and $Z$ are dependent on the definition of the axis assigned to the cross-section. In the present study, we pay attention to the two different axis configurations as indicated in Figure 1b, with respect to which the cross-sectional performance is examined. We shall find that $I$ (and $R_{g}$ ) takes the identical value for the two axes, even when the axis is rotated by an arbitrary angle in the same plane, because of the four-fold symmetry of the cross-section to be considered; see Appendix $C$ for details.

The effect of geometric variation on $R_{g}$ can be addressed by introducing the improvement ratio, $\eta$, defined by:

$$
\eta=\frac{R_{g w}-R_{g w}^{0}}{R_{g w}^{0}} .
$$

Here, $R_{g w}$ is the gyration radius of the woody portion sandwiched by two concentrically arranged rounded squares; see Figure $1 \mathrm{~b}$. $R_{g w}^{0}$ is the gyration radius of an annulus, obtained by $\theta_{\text {inn }}=\theta_{\text {out }}=$ $\pi / 4$, having the same area as that of the rounded square-shaped woody portion. The improvement ratio $\eta$ measures how $R_{g w}$ increases compared with the case of a simple annulus. When $\eta$ takes a large absolute value with a positive (or negative) sign, it means that the gyration radius has increased (decreased) due to the change in cross-section shape from the simple annulus to a rounded square.

Similarly, we also define the improvement ratio of the section modulus of the system, $\zeta$, defined by:

$$
\zeta=\frac{Z_{w}-Z_{w}^{0}}{Z_{w}^{0}}
$$

Similar to the case of gyration radius, $Z_{w}$ is the section modulus of the woody portion, and $Z_{w}^{0}$ is that of an annulus having the same area as the woody portion. Considering the dependence of $Z_{w}$ on the direction of the axis, as well as taking into account the four-fold symmetry of the cross-sectional shape, we chose the specific two axis configurations, labeled as Axis-1 and Axis-2, which are illustrated in Figure 1b.

\section{Numerical Conditions}

Each of the two concentric rounded squares has a set of four geometric parameters: $\left\{\ell_{\text {out }}, \theta_{\text {out }}, h_{\text {out }}, a_{\text {out }}\right\}$ for the outer square and $\left\{\ell_{\text {inn }}, \theta_{\text {inn }}, h_{\text {inn }}, a_{\text {inn }}\right\}$ for the inner square. For both squares, the areas $A_{\text {out }}=\pi a_{\text {out }}^{2}$ and $A_{\text {inn }}=\pi a_{\text {inn }}^{2}$ are set to be unchanged in order for the area between 
the two concentric squares, $A_{\text {out }}-A_{\text {inn }}$, to remain constant, as well. These constant-area conditions make each of $h_{\text {out }}$ and $h_{\text {inn }}$ dependent on the remaining three parameters, respectively.

In the following discussion, the relative ratio of $a_{\text {out }}$ to $a_{\text {inn }}$ is set to $a_{\text {out }} / a_{\text {inn }}=1.5$, as a case study, without loss of generality of our conclusion, while $a_{\text {inn }}$ will be used as the unit of length.

\section{Results: Improvement Ratio}

To examine the cross-sectional performance of the system, we changed the values of $\ell_{\text {out }}, \ell_{\text {inn }}$, $\theta_{\text {out }}$, and $\theta_{\text {inn }}$ in a systematic manner to obtain the geometric dependences of $\eta$ and $\zeta$, only a part of which will be shown below.

Figure 4a shows the improvement ratio $\eta$ as a function of $\theta_{\text {out }}$ and $\theta_{\text {inn }}$. Other geometric parameters were set to be $\ell_{\text {out }}=1.2$ and $\ell_{\text {inn }}=0.8$ in units of $a_{\text {inn }}$. It was observed that $\eta$ grew with $\theta_{\text {out }}$ in a nearly parabolic manner without a local maximum peak for every choice of $\theta_{\text {inn, }}$ and the effect of $\theta_{\text {inn }}$ variation was insignificant under the present condition. The maximum value of $\eta$ could be obtained at $\theta_{\text {out }}=\pi / 2$ and $\theta_{\text {inn }}=\pi / 4$, which corresponded to the hollow cross-section composed of a quadrangular-shaped outer curve and a circular-shaped inner curve. We saw that the maximum increment in $\eta$ was a few percent at most, which will be intuitively understood by an analytic estimation discussed later.
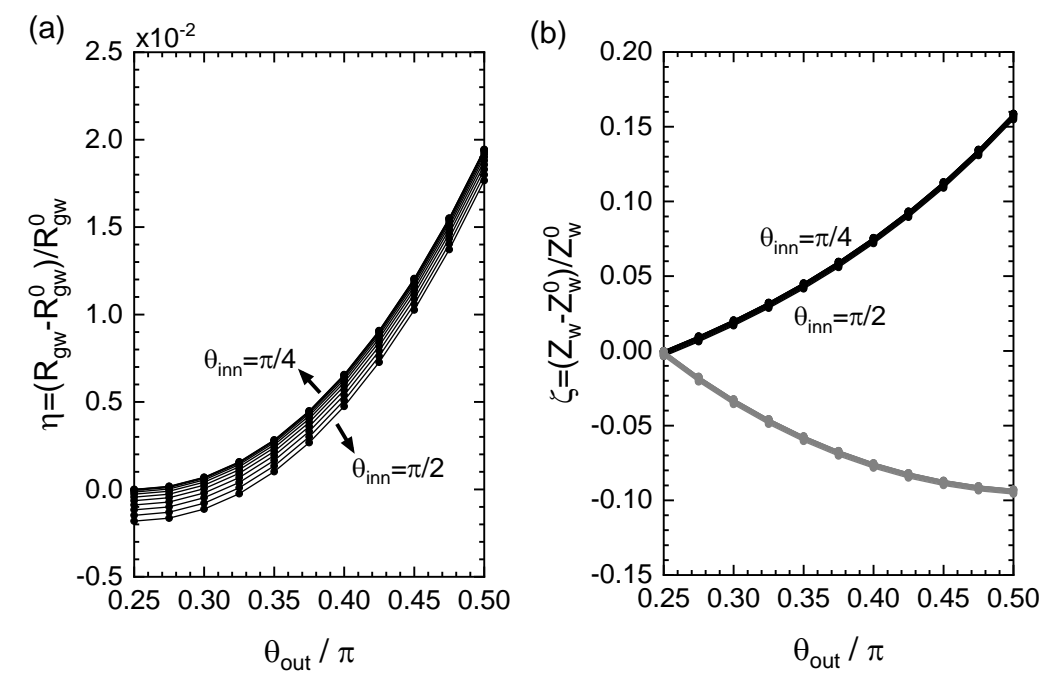

Figure 4. Improvement ratios, $\eta$ and $\zeta$, for the case of $\ell_{\text {out }} / a_{\text {out }}=1.2$ and $\ell_{\text {inn }} / a_{\text {inn }}=0.8$. (a) $\eta$ as a function of $\theta_{\text {out }}$. (b) $\zeta$ as a function of $\theta_{\text {out }}$. Upper (black) and lower (gray) branches correspond to the results with respect to Axis-1 and Axis-2 depicted in Figure 1b, respectively.

Figure $4 \mathrm{~b}$ shows the improvement ratio $\zeta$ for the section modulus. The upper branches (almost all of the curves with different $\theta_{\text {inn }}$ appear to be superimposed) correspond to the results with respect to Axis-1, and the lower branches to Axis-2. In the upper branches, a similar trend as that of $\eta$ was observed, while the insensitivity to the change in $\theta_{\text {inn }}$ was more prominent. The increment obtained at the maximum was estimated to be 16 percent, which would give a feasible contribution to the mechanics of the hollow columns. This increment was attributed to the elongation of the distance $e$ from the axis to the extreme edge of the outer boundary, which was a direct consequence of the geometric variation in the cross-section from the simple circle to a rounded square.

If the magnitude relation between $\ell_{\text {out }}$ and $\ell_{\text {inn }}$ was altered, the maximum increments in $\eta$ and $\zeta$ were both depressed. An example is shown in Figure 5, in which $\ell_{\text {out }}=0.8$ and $\ell_{\text {inn }}=1.2$ were set in units of $a_{\mathrm{inn}}$. It thus turned out that, to obtain a greater $\eta$ and $\zeta$, it was advantageous to make the outer curve more square while making the inner curve more circular. 

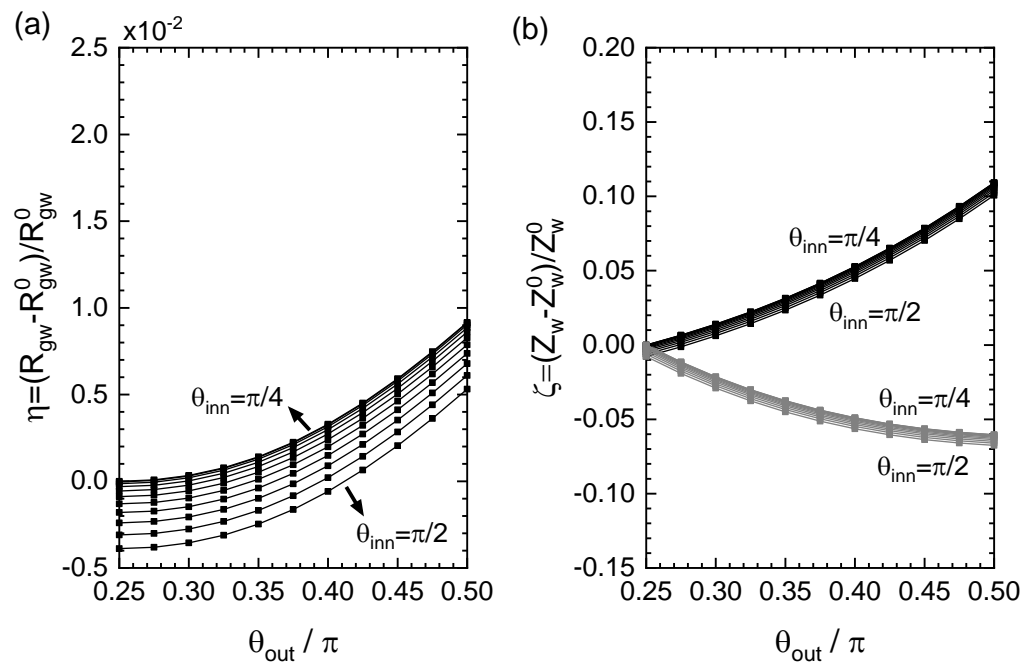

Figure 5. Improvement ratios for the case of $\ell_{\text {out }} / a_{\text {out }}=0.8$ and $\ell_{\text {inn }} / a_{\text {inn }}=1.2$. (a) $\eta ;$ (b) $\zeta$.

\section{Discussion}

\subsection{Variable Range Estimation of $\eta$ and $\zeta$}

Given the ratio of $a_{\text {out }} / a_{\text {inn }}$, the maximum and minimum values of $\eta$ could be roughly estimated by considering the following two extreme situations: (i) the outer curve is an exact quadrangular square with the enclosed area of $\pi a_{\text {out }}^{2}$ and the inner curve is a simple circle with the enclosed area of $\pi a_{\mathrm{inn}}^{2}$; and (ii) vice versa (i.e., the outer circular curve and the inner quadrangular curve with the definitions of the areas same as (i)). Suppose that $a_{\text {out }}=p a_{\text {inn }}$ with a proportional constant $p$. We then have:

$$
\eta=\sqrt{\frac{(\pi / 3) p^{4}-1}{p^{4}-1}}-1 \text { for case (i), }
$$

and

$$
\eta=\sqrt{\frac{p^{4}-(\pi / 3)}{p^{4}-1}}-1 \text { for case (ii). }
$$

Substituting $p=1.5$ in accord with our numerical condition in Sec.IV, we obtain $\eta \simeq 0.03$ for Case (i) and $\eta \simeq-0.006$ for Case (ii). Therefore, $\eta$ should take an intermediate value between the two extrema, as was consistent with our numerical results shown in Figures $4 \mathrm{a}$ and $5 \mathrm{a}$. A similar argument holds true for the maximum and minimum values of $\zeta$.

\subsection{Effect of the Fillet at the Vertices}

It was proven that the cross-sectional performance was highest when the outer boundary was a quadrangle with sharpened vertices and the inner boundary was a circle [27]. In practice, however, a strict quadrangle is not preferred as the outer boundary. This is because when the hollow prism is subjected to bending deformation, stress concentrations occur around acute vertices so that it locally breaks. To prevent the local breaking, it is better to fillet corners as suggested in the present model. We demonstrated that such filleted corners did not significantly reduce the cross-sectional performance of the hollow square prism, thus achieving the trade-off between the ideal performance obtained by sharpening the corners at the outer boundary and the suppression of the fragility at the corners realized by rounding them. This may be the wisdom of wild plants, while a certain physiological reason should also be possible for the preference of filleted corners.

It also should be noted that, even if the corners were filleted to some extent, the bending stress may be concentrated at the corner, causing premature failure. In order to clarify the stress distribution 
near the corners quantitatively, it is necessary to analyze the effect of stress triaxiality or perform finite element modeling, which is an interesting problem left for the future.

\subsection{Implication for the Mechanical Rigidity and Strength}

The relationship between cross-sectional shape and column mechanical stability and strength may need to be explained in more detail for readers outside the field. In structural mechanics, the gyration radius of the area, $R_{g}$, is known as a key quantity for comparing the buckling resistance of elastic columns with different cross-sectional shapes. Long columns often buckle when they receive an axial stress that exceeds a certain threshold value. The maximum compressive stress the column can withstand is called the buckling stress. An important fact is that the magnitude of the buckling stress is determined by $R_{g}$; more concretely, the larger $R_{g}$, the greater the buckling stress. The Euler column formula can be used to analyze the buckling of a long column with a load applied along the central axis:

$$
\sigma_{\mathrm{cr}}=\frac{\pi^{2} E}{c\left(L / R_{g}\right)^{2}}
$$

In Equation (7), $\sigma_{\mathrm{cr}}$ is the critical stress for the column to buckle, $E$ is the Young modulus of the material, $L$ is the column length, and the constant $c$ accounts for the end conditions of the column. The formula indicates that, in order to obtain a column structure that can withstand a large compressive stress, it is necessary to increase $R_{g}$. Our results proved that imparting convex rounded sides and filleted corners to the cross-section did not significantly degrade the large buckling resistance shown by strict rectangular square columns endowed with the maximum value of $R_{g}$.

The section modulus, $Z$, is another key quantity for considering the mechanical stability of elastic columns. $Z$ measures the strength of a column; the higher the section modulus, the higher will be the resistance to yield under bending. By multiplying $Z$ with the yield strength $\sigma_{y}$ of the constituent material of the column, we can calculate the upper limit bending moment, $M_{y}=Z \sigma_{y}$, that the column can withstand without plastic deformation. Our results showed that the rounded square cross-section was beneficial for securing a large value of $M_{y}$ compared with the case of a circular cross-section, which may be one of the reasons why a kind of plant stem exhibits the rounded square cross-sections.

It is also noteworthy that the second moment of area, $I$, is also important for evaluating the cross-sectional performance. It represents the degree of bending stiffness of a column. Multiplying $I$ by $E$ of the constituent material, we obtained the bending stiffness $E I$ of the column. Since in the present work, the area of cross-section $A$ was fixed, the relation of $I \propto R_{g}^{2}$ holds for every system under consideration; in this case, the effect of $I$ on the cross-sectional performance can be deduced easily from that result of $R_{g}$.

\subsection{Versatility of the Theoretical Model}

As a closing remark, we mention the scope of application of our mathematical model to actual wild plants. When you observe culms and stems of actual plants, you will find that the shape of the cross-section is not uniform, but gradually changes along the height direction. Therefore, the morphological quantities that characterize the cross-sectional performance of the plants, such as $A, I, R_{g}$, and $Z$, generally change depending on the height from the ground. Our model was effective at dealing with such problems. In fact, the theoretical model developed in this study could change the cross-sectional shape continuously by properly controlling the parameter values. Therefore, by collecting the actual measurement data of the cross-section of the plant and reproducing the shape of each cross-section with different heights by the model, it was possible to estimate the change in cross-sectional performance along the height direction accurately. This made it possible to analyze the three-dimensional mechanical behavior of hollow columnar plants with a polygonal cross-section, such as square bamboo.

It also should be emphasized that the model we developed could be extended to triangles, pentagons, hexagons, and other arbitrary polygons in a straightforward manner. This is realized by 
replacing the reference square depicted in Figure 2 with another kind of polygon, followed by the same drawing procedure of long and short arcs. Figure 6a shows an example of a rounded triangle, whose shape looks similar to the cross-section of a papyrus (Cyperus microiria) given in Figure 1d. Furthermore, it is possible to break the discrete rotational symmetry of the model, by setting a different value of $\theta$ at each vertex. Figure $6 \mathrm{~b}$ shows such an example of a rounded square with no rotational symmetry. By extending this model in the above-mentioned way, it becomes possible to reproduce more faithfully the cross-section of polygonal stems, culms, and branches of actual plants whose shape should vary considerably from sample to sample in general. In future work, we plan to measure the cross-sectional shape of square bamboo in detail and reproduce it numerically using the present model.

(a)

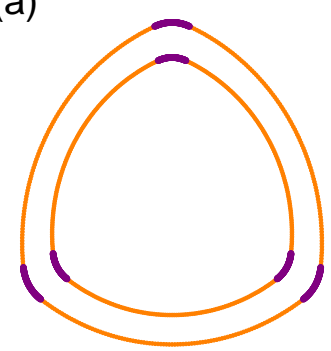

(b)

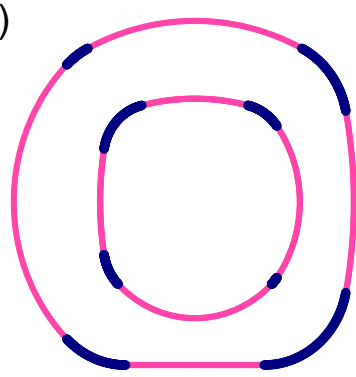

Figure 6. (a) A model of a triangular cross-section with rounded sides and filleted corners, mimicking the cross-section of a papyrus; see Figure 1. (b) A model of a non-symmetric hollow square cross-section.

\section{Conclusions}

In this article, we proposed a new mathematical model of a quadrilateral consisting of arched edges and filleted vertices. This model could accurately reproduce the cross-sectional shape of plants with hollow prismatic structures such as square bamboo, perilla, and cyperus. Therefore, the model was very useful when investigating the mechanical properties of plants and when designing mechanical optimal structures that mimic the functional morphology of plants. As an application example, we showed an analytical solution of the gyration radius and the section modulus of the area of a hollow square column whose cross-sectional shape resembled that of a square bamboo. The proposed model could be applied to various polygons such as triangles, pentagons, hexagons, as well as rectangles. In addition, an asymmetrical polygonal cross-section could be reproduced with a slight expansion. We hope that the model will be used as an analysis tool to reproduce the morphology of actual plants with high accuracy.

Author Contributions: Conceptualization, H.S., A.I., and M.S.; methodology, H.S.; formal analysis, H.S., N.F., and Y.K.; writing, original draft preparation, H.S.; funding acquisition, H.S., A.I., and M.S. All authors read and agreed to the published version of the manuscript.

Funding: This work was supported by JSPS KAKENHI Grant Numbers 18H03818, 18H02244, 18KT0037, 19K03766, and $19 \mathrm{H} 05359$.

Acknowledgments: We would like to thank T. Fukuhara and N. Matsuoka for providing us with cross-sectional photographs of the plants.

Conflicts of Interest: The authors declare no conflict of interest.

\section{Appendix A}

Appendices A and B are devoted to derive the analytic expression of the second moment of area, $I$, of a square with rounded edges and fillet corners. Once $I$ is obtained, the gyration radius $R_{g}$ and the section modulus $Z$ of the same cross-section are easily obtained. The following discussion presents some preparatory material for deriving the area second moment formula used in Appendix B. 
For a given cross-section spanned in the $x$ - $y$ coordinate plane, the first moment of area, $F_{k}$, and the second moment of area, $I_{k}$, with respect to the $k$ axis are respectively defined by:

$$
\begin{aligned}
I_{k} & =\iint k^{2} d x d y, \quad k=x, y, \\
F_{k} & =\iint k d x d y, \quad k=x, y .
\end{aligned}
$$

It is easily derived from the definitions that the second moment of area $I_{X}$ with respect to the $X$ axis, which is obtained by translating the $x$ axis by $y_{0}$ in the vertical direction, is represented as:

$$
I_{X}=I_{x}-y_{0} F_{x}+y_{0}^{2} A,
$$

where $A$ is the area of the cross-section considered. This formula will be used in Appendix B.

For later use, two specific kinds of geometry are considered: one is the circular sector depicted in Figure A1a, and the other is the right triangle depicted in Figure A1b. The geometry of the circular sector is uniquely determined by the radius $r$ and the two angles, $\alpha$ and $\beta$. Hence, the second and first moments of the area of the sector with respect to the $k$-axis $(k=x, y)$ are given by:

$$
\begin{aligned}
& I_{x}^{c}(r, \alpha, \beta)=\frac{\rho^{4}}{8}\left[(\beta-\alpha)-\frac{\sin 2 \beta-\sin 2 \alpha}{2}\right], \\
& I_{y}^{c}(r, \alpha, \beta)=\frac{\rho^{4}}{8}\left[(\beta-\alpha)+\frac{\sin 2 \beta-\sin 2 \alpha}{2}\right]
\end{aligned}
$$

and:

$$
\begin{aligned}
& F_{x}^{c}(r, \alpha, \beta)=\frac{\rho^{3}}{3}(-\cos \beta+\cos \alpha), \\
& F_{y}^{c}(r, \alpha, \beta)=\frac{\rho^{3}}{3}(\sin \beta-\sin \alpha),
\end{aligned}
$$

respectively. The superscript $c$ indicates that the quantity is associated with the circular sector.

Similarly, the geometry of the right triangle is uniquely determined by the base length $d$ and the apex angle $\phi$. Hence, we have for the triangle,

$$
\begin{aligned}
I_{x}^{t}(d, \phi) & =\frac{d^{4}}{12} \tan \phi, \quad I_{y}^{t}(d, \phi)=\frac{d^{4}}{4} \tan \phi, \\
F_{x}^{t}(d, \phi) & =\frac{d^{3}}{6} \tan \phi, \quad F_{x}^{t}(d, \phi)=\frac{d^{3}}{3} \tan \phi .
\end{aligned}
$$

The superscript $t$ indicates that the quantity is associated with the triangle.

(a)

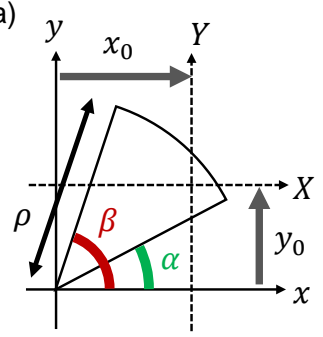

(b)

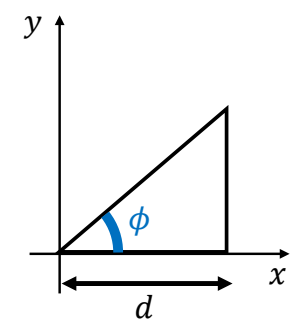

Figure A1. (a) Circular sector defined by the angles $\alpha$ and $\beta$ and the radius $\rho$. (b) Right-angle triangle with the base $d$ and the apex $\phi$. 


\section{Appendix B}

Our immediate goal is to formulate the second moment of area of the rounded-corner squared cross-section, defined by the previous section, with respect to the $x$-axis. To this aim, we decompose the first quadrant part of the cross-section into the four domains as illustrated by Figure A2. For the four domains, the second moments of the area with respect to the $x$ axis are written by $J_{i}(i=1,2,3,4)$; then, the second moment of the whole square, $J_{\text {all }}$, is given by:

$$
J_{\text {all }}=4\left(J_{1}+J_{2}+J_{3}-J_{4}\right) .
$$

In the following, we will show how to calculate the four components $J_{i}(i=1,2,3,4)$ in order.

(a)

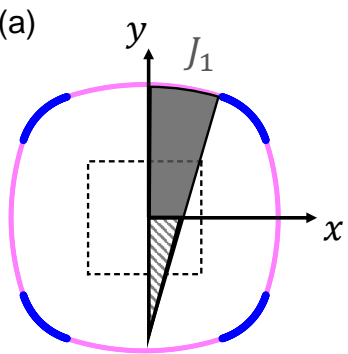

(b)

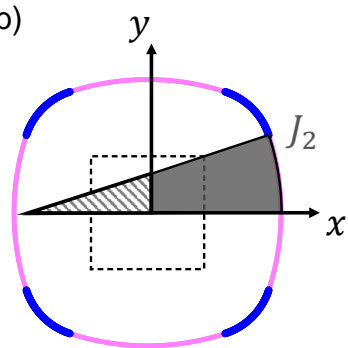

(c)

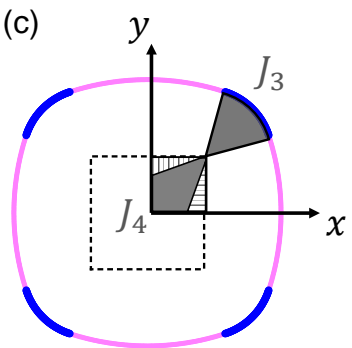

Figure A2. Diagram of the four domains in the first quadrant $(x>0$ and $y>0)$ of the rounded square. Each domain is marked by a gray area. $J_{i}(i=1,2,3,4)$ represents the second moment of area of the $i$ th domain with respect to the $x$ axis. (a) Domain 1 ; the gray area obtained by excluding the shaded right triangle from the vertically elongated sector. (b) Domain 2; the gray area obtained by excluding the triangle from the long sector. (c) Domain 3 (the gray small sector at the upper right) and Domain 4 (the gray kite-shaped square obtained by excluding the two shaded triangles from the right square).

For Domain 1 , the second moment of area with respect to the $x$-axis is given by:

$$
J_{1}=J_{1 X}^{c}-J_{1 X}^{t}
$$

where $J_{1 X}^{c}$ is the second moment of the circular sector which is elongated in the $x$ direction, as depicted in Figure A2a, and $J_{1 X}^{t}$ is that of the right triangle (shaded region) elongated in the $x$ direction, as well. The explicit forms of them read:

$$
\begin{aligned}
& J_{1 X}^{c}=I_{x}^{c}\left(\rho_{1}, \alpha_{1}, \beta_{1}\right)-2 d F_{x}^{c}\left(\rho_{1}, \alpha_{1}, \beta_{1}\right)+d_{1}^{2} A_{1}^{c}, \\
& J_{1 X}^{t}=I_{y}^{t}\left(d_{1}, \phi_{1}\right)-2 d F_{y}^{t}\left(d_{1}, \phi_{1}\right)+d_{1}^{2} A_{1}^{t} .
\end{aligned}
$$

Here, the subscript 1 attached to the arguments indicates that their value is equal to those listed in the row of "Domain 1" in Table A1. $A_{1}^{c}$ and $A_{1}^{t}$ are the area of the circular sector and the triangle, respectively, given by $A_{1}^{c}=\left(\phi_{1} / 2\right) \rho_{1}^{2}$ and $A_{1}^{t}=\left(d_{1}^{2} / 2\right) \tan [(\pi / 2)-\theta]$. The only exception is the case in which $\theta=\pi / 2$. In this case, both $\rho_{1}$ and $d_{1}$ diverge, so that Equation (A11) does not work properly. Instead, we should use the different expression of $J_{1}=(\ell / 6)[(\ell / 2)+h]^{3}$, only when $\theta=\pi / 2$.

For Domain 2, the second moment of area, $J_{2}$, is given by:

$$
J_{2}=I_{x}^{c}\left(\rho_{2}, \alpha_{2}, \beta_{2}\right)-I_{x}^{t}\left(d_{2}, \phi_{2}\right),
$$

for $\pi / 4 \leq \theta<\pi / 2$. Only when $\theta=\pi / 2$, it is replaced by $J_{2}=\left(\ell^{3} / 24\right)[(\ell / 2)+h]$.

For Domain 3, we have:

$$
J_{3}=I_{x}^{c}\left(\rho_{3}, \alpha_{3}, \beta_{3}\right)+\ell F_{x}^{c}\left(\rho_{3}, \alpha_{3}, \beta_{3}\right)+\left(\frac{\ell}{2}\right)^{2} A_{3}^{c},
$$


where $A_{3}^{c}=\left(\rho_{3}^{2} / 2\right)\left(\beta_{3}-\alpha_{3}\right)$. This expression applies regardless of the value of $\theta$, as long as $\pi / 4 \leq$ $\theta \leq \pi / 2$.

For Domain 4, we have:

$$
J_{4}=\frac{\ell^{4}}{48}-\left(J_{4 \mathrm{~V}}^{t}+J_{4 \mathrm{H}}^{t}\right) .
$$

Here, $J_{4 \mathrm{~V}}^{t}$ is the second moment of area of the vertically elongated triangle, and $J_{4 \mathrm{H}}^{t}$ is that of the horizontally elongated triangle, both of which are shaded in Figure A2c. They are explicitly written as:

$$
\begin{aligned}
& J_{4 \mathrm{~V}}^{t}=I_{y}^{t}\left(d_{4}, \phi_{4}\right)-\ell F_{y}^{t}\left(d_{4}, \phi_{4}\right)+\left(\frac{\ell}{2}\right)^{2} A_{4}^{t}, \\
& J_{4 \mathrm{H}}^{t}=I_{x}^{t}\left(d_{4}, \phi_{4}\right)-\ell F_{x}^{t}\left(d_{4}, \phi_{4}\right)+\left(\frac{\ell}{2}\right)^{2} A_{4}^{t},
\end{aligned}
$$

where $A_{4}^{t}=\left(\ell^{2} / 8\right) \tan \phi_{4}$ is the area of the shaded triangle.

Table A1. Parameter settings for the four domains.

\begin{tabular}{cccccc}
\hline Parameters & $\rho$ & $\alpha$ & $\beta$ & $d$ & $\phi$ \\
\hline Domain 1 & $\frac{\ell}{2 \cos \theta}+h$ & $\theta$ & $\frac{\pi}{2}$ & $\frac{\ell}{2}(\tan \theta-1)$ & $\frac{\pi}{2}-\theta$ \\
Domain 2 & $\frac{\ell}{2 \cos \theta}+h$ & 0 & $\frac{\pi}{2}-\theta$ & $\frac{\ell}{2}(\tan \theta-1)$ & $\frac{\pi}{2}-\theta$ \\
Domain 3 & $h$ & $\frac{\pi}{2}-\theta$ & $\theta$ & $\mathrm{n} / \mathrm{a}$ & $\mathrm{n} / \mathrm{a}$ \\
Domain 4 & $\mathrm{n} / \mathrm{a}$ & $\mathrm{n} / \mathrm{a}$ & $\mathrm{n} / \mathrm{a}$ & $\frac{\ell}{2}$ & $\frac{\pi}{2}-\theta$ \\
\hline
\end{tabular}

\section{Appendix C}

This proves that the value of $I$ is uniquely determined only by the shape of the cross-section, regardless of the axial choice, when the cross-section is four-fold symmetric.

Suppose that the coordinate axes in the original $x-y$ plane are mutually rotated by the angle $\varphi$. The new coordinates are defined by:

$$
\begin{aligned}
& u=x \cos \varphi+y \sin \varphi, \\
& v=-x \sin \varphi+y \cos \varphi .
\end{aligned}
$$

The second moments of area with respect to the rotated axes read:

$$
\begin{aligned}
& I_{u}=I_{x} \cos ^{2} \varphi+I_{y} \sin ^{2} \varphi-I_{x y} \sin 2 \varphi, \\
& I_{v}=I_{x} \sin ^{2} \varphi+I_{y} \cos ^{2} \varphi+I_{x y} \sin 2 \varphi,
\end{aligned}
$$

where $I_{x y}$ is defined by:

$$
I_{x y}=\iint x y d x d y
$$

We remind that when the cross-section to be considered is endowed with a four-fold symmetry in the $x-y$ plane, we have:

$$
I_{x}=I_{y} \text { and } I_{x y}=0 .
$$

Substituting them into Equations (A18) and (A19), we achieve the conclusion that:

$$
I_{x}=I_{u}=I_{v}=I_{y},
$$


which holds true for arbitrary $\varphi$. This result indicates that the second moment of the four-fold symmetric cross-sectional area does not depend on the rotation angle of the axis and is uniquely determined by the cross-sectional shape.

\section{References}

1. Galileo, G. Discorsi e Dimostrazioni Matematiche Intorno a Due Nuove Scienze Attenenti Alla Meccanica e $i$ Movimenti Locali; Elzeviro, L., Ed.; Appresso gli Elsevirii: Leida, The Netherlands, 1638.

2. Shima, H.; Sato, M.; Inoue, A. Self-adaptive formation of uneven node spacings in wild bamboo. Phys. Rev. E 2016, 93, 022406. [CrossRef] [PubMed]

3. Sato, M.; Inoue, A.; Shima, H. Bamboo-inspired optimal design for functionally graded hollow cylinders. PLoS ONE 2017, 12, e0175029. [CrossRef] [PubMed]

4. Scurlock, J.M.O.; Dayton, D.C.; Hames, B. Bamboo: An overlooked biomass resource? Biomass Bioenergy 2000, 19, 229-244. [CrossRef]

5. Inoue, A.; Sato, M.; Shima, H. Maximum size-density relationship in bamboo forests: Case study of Phyllostachys pubescens forests in Japan. For. Ecol. Manag. 2018, 425, 138-144. [CrossRef]

6. Inoue, A.; Shimada, M.; Sato, M.; Shima, H. Estimation of culm volume reduction factors in five bamboo species (Phyllostachys spp.). J. For. Res. 2019, 30, 2069-2078. [CrossRef]

7. Song, X.; Peng, C.; Zhou, G.; Gu, H.; Li, Q.; Zhang, C. Dynamic allocation and transfer of non-structural carbohydrate, a possible mechanism for the explosive growth of moso bamboo (Phyllostachys heterocycle). Sci. Rep. 2016, 6, 25908. [CrossRef] [PubMed]

8. Yen, T.M. Culm height development, biomass accumulation and carbon storage in an initial growth stage for a fast-growing moso bamboo (Phyllostachys pubescens). Bot. Stud. 2016, 57, 10. [CrossRef] [PubMed]

9. Farrelly, D. The Book of Bamboo; Sierra Club Books: San Francisco, CA, USA, 1984.

10. Inoue, A.; Koshikawa, K.; Sato, M.; Shima, H. Allometric equations for predicting the aboveground biomass of square bamboo, Chimonobambusa quadrangularis. J. For. Res. 2019, 24, 376-381. [CrossRef]

11. Gielis, J. The Geometrical Beauty of Plants; Atlantis Press: Paris, France, 2017.

12. A Photo Taken from FUKUHARA's Website. Available online: https://ww1.fukuoka-edu.ac.jp/ fukuhara/ keitai/4-2.html (accessed on 21 May 2020).

13. A Photo Taken from MATSUMOMUSHI's Website. Available online: http://plants.minibird.jp/ hydrophytes/plants/shissei/sa_gyou/sankakui/sankakui.html (accessed on 21 May 2020).

14. Lamé, G. Examen de Differentes Méthodes Employées Pour Résoudre les Problèmes de Géometrie; M. V. Courcier Imprimeur Libraire: Paris, France, 1818.

15. Shi, P.J.; Huang, J.G.; Hui, C.; Grissino-Mayer, H.D.; Tardif, J.C.; Zhai, L.H.; Wang, F.S.; Li, B.L. Capturing spiral radial growth of conifers using the superellipse to model tree-ring geometric shape. Front. Plant Sci. 2015, 6, 856. [CrossRef] [PubMed]

16. Gielis, J. A generic geometric transformation that unifies a wide range of natural and abstract shapes. Am. J. Bot. 2003, 90, 333-338. [CrossRef] [PubMed]

17. Gielis, J.; Caratelli, D.; Fougerolle, Y.; Ricci, P.E.; Tavkelidze, I.; Gerats, T. Universal natural shapes: From unifying shape description to simple methods for shape analysis and boundary value problems. PLOS ONE 2012, 7, e3974968. [CrossRef]

18. Shi, P.; Ratkowsky, D.A.; Gielis, J. The generalized Gielis geometric equation and its application. Symmetry 2020, 12, 645. [CrossRef]

19. Jafari, M.; Chaleshtari, M.H.B.; Abdolalian, H.; Craciun, E.M.; Feo, L. Determination of forces and moments per unit length in symmetric exponential FG plates with a quasi-triangular hole. Symmetry 2020, 12, 834. [CrossRef]

20. Klingenberg, C. Analyzing fluctuating asymmetry with geometric morphometrics: Concepts, methods, and applications. Symmetry 2015, 7, 843-934. [CrossRef]

21. Schmidt, D.; Kahlen, K. Towards more realistic leaf shapes in functional-structural plant models. Symmetry 2018, 10, 278. [CrossRef]

22. Shi, P.; Zheng, X.; Ratkowsky, D.; Li, Y.; Wang, P.; Cheng, L. A simple method for measuring the bilateral symmetry of leaves. Symmetry 2018, 10, 118. [CrossRef]

23. Cao, L.; Shi, P.J.; Li, L.; Chen, G. A new flexible sigmoidal growth model. Symmetry 2019, 11, 204. [CrossRef] 
24. Gielis, J.; Caratelli, D.; Shi, P.; Ricci, P.E. A note on spirals and curvature. Growth Form 2020, 1, 1-8. [CrossRef] 25. Dargahi, M.; Newson, T.; Moore, J. Buckling behaviour of trees under self-weight loading. Forestry 2019, 92, 393-405. [CrossRef]

26. Fiorello, I.; Dottore, E.D.; Tramacere, F.; Mazzolai, B. Taking inspiration from climbing plants: methodologies and benchmarks-A review. Bioinspir. Biomim. 2020, 15, 031001. [CrossRef] [PubMed]

27. Gere, J.M.; Timoshenko, S.P. Mechanics of Materials; Van Nostrand Reinhold Company: New York, NY, USA, 1972.

(C) 2020 by the authors. Licensee MDPI, Basel, Switzerland. This article is an open access article distributed under the terms and conditions of the Creative Commons Attribution (CC BY) license (http:/ / creativecommons.org/licenses/by/4.0/). 